\title{
Pierre Laforgue, Stendhal alla Monaca. Le romantisme, le romanesque, le roman
}

Paris, Classiques Garnier, 2016, 402 p.

\section{Laure Lévêque}

\section{OpenEdition}

\section{Journals}

Édition électronique

URL : http://journals.openedition.org/recherchestravaux/915

DOI : 10.4000/recherchestravaux.915

ISSN : 1969-6434

Éditeur

UGA Éditions/Université Grenoble Alpes

Édition imprimée

ISBN : 978-2-37747-006-8

ISSN : 0151-1874

\section{Référence électronique}

Laure Lévêque, "Pierre Laforgue, Stendhal alla Monaca. Le romantisme, le romanesque, le roman »,

Recherches \& Travaux [En ligne], 90 | 2017, mis en ligne le 01 juin 2017, consulté le 25 septembre 2020.

URL : http://journals.openedition.org/recherchestravaux/915 ; DOI : https://doi.org/10.4000/

recherchestravaux.915

Ce document a été généré automatiquement le 25 septembre 2020

(C) Recherches \& Travaux 


\title{
Pierre Laforgue, Stendhal alla Monaca. Le romantisme, le romanesque, le roman
}

\author{
Paris, Classiques Garnier, 2016, 402 p.
}

\author{
Laure Lévêque
}

\section{RÉFÉRENCE}

Pierre Laforgue, Stendhal alla Monaca. Le romantisme, le romanesque, le roman, Paris, Classiques Garnier, 2016, 402 p.

1 Une métaphore ingénieuse, venue de la Chartreuse, sert de point de départ à la réflexion et fournit au volume son titre énigmatique, thématiquement bien accordé à la Chartreuse, corpus privilégié de l'investigation, avec Le Rouge et le Noir, que viennent compléter les Chroniques italiennes, Rome, Naples et Florence en 1817 et Racine et Shakespeare.

2 Stendhal alla monaca, c'est Stendhal chiffré, Stendhal cryptique que Pierre Laforgue se propose de déchiffrer pour son lecteur, partant de ce que la démarche cryptique suppose, chez Stendhal, une "défiance à l'égard du langage» (p.12) que Pierre Laforgue corrèle à la dimension philosophique qu'assume le romantisme, à la fois « crise de la représentation et du sens » (p. 19).

3 Après avoir fait justice d'un Stendhal estampillé « réaliste » qu'on ne croyait pourtant plus guère de mise, Pierre Laforgue entend donc s'engager sur la voie d'une « lecture alla monaca », entreprise dont la difficulté tient à ce que, à la différence de Fabrice qui reçoit le « grand code » de la Sanseverina, ni le lecteur ni Pierre Laforgue, ne l'ont, eux, reçu de Stendhal, au risque de voir proliférer diverses interprétations hasardées.

4 Ainsi, lorsque l'auteur en vient à l'explicitation du sous-titre - « le romantisme, le romanesque, le roman »-, approximations et schématisme éclatent et se donne cours une argumentation passablement anachronique comme Pierre Laforgue remonte la 
piste d'un "romanesque critique ", dès longtemps frayée (p. 19). Sur cette notion de romanesque, certains présupposés déconcertent : en quoi l'épisode de la note secrète du Rouge devrait-il être exempté de romanesque (p. 20) alors même qu'il traite des préparatifs d'un coup d'État? Règne là un certain sfumato, qui n'appartient pas qu'au Corrège. Pierre Laforgue rappelle ainsi des évidences quand il revient sur l'interpénétration, dans Lucien Leuwen, d'un romanesque de type courtois avec son envers, dégradé. Comme l'auteur le dit lui-même plus loin à propos d'autres enjeux, "l'affaire depuis longtemps est entendue »(p. 22). De même, rejeter Flaubert du côté de l'exigence réaliste, lui qui s'en est si constamment défendu, peut au moins étonner. Et pareillement le rejet du romantisme qui lui est ici prêté, comme si le romantisme ne donnait pas lieu, au fil du long $\mathrm{XIX}^{\mathrm{e}}$ siècle, à de multiples réinvestissements. Ces questions ne vont pas de soi et, sauf à faire l'objet de définitions serrées - ce qui n'est pas le cas ici-, ne sauraient être résolues à coup de formules volontaristes car, de même que Laforgue s'étonne d'un front qui milite contre un Stendhal romantique, ses propres positions sur l'œuvre flaubertienne - réaliste et non romantique -, ne sont pas forcément des universaux.

Que Stendhal se sépare du romantisme du premier Hugo et qu'il se réclame du romanticisme n'est pas exactement une révélation. Et pas davantage la bataille à fronts renversés qui, dans les années 1820 , oppose des romantiques conservateurs en politique à des libéraux classiques en littérature. La présence de notes renvoyant aux travaux qui ont précédé ceux de l'auteur aurait sans doute permis de resituer ces questions dans une perspective diachronique indispensable à la compréhension de ces enjeux et d'avancer plus sereinement sur un terrain désormais bien déminé. Ce qui aurait été cohérent avec la pétition de principe dont se revendique Pierre Laforgue et avec l'idée de la recherche qu'il entend défendre en replaçant ses travaux dans « un parcours qui s'inscrit dans le temps et qui en porte la marque» (p. 35). C'est courir autrement le risque du "pêle-mêle", pour reprendre le terme même de Pierre Laforgue (p. 24), gros de distorsions, et de toutes les anamorphoses.

6 Le recueil est organisé en fonction des trois sections délimitées par le sous-titre, «le romantisme, le romanesque, le roman ».

7 La première partie, "le romantisme", composée de cinq articles, envisage ainsi successivement: le rôle de l'Italie dans l'élaboration d'une pensée de la liberté chez Stendhal, qui puisse s'acclimater tant en France qu'en Italie ("Viva la libertà... »); la place génétique qu'occupent les deux libelles de 1823 et 1825 (Racine et Shakespeare) dans l'histoire de la notion, déterminant le contenu que lui assignera Hugo au moment de la bataille d'Hernani (« Du romanticisme au romantisme »...) ; les jugements de valeur qui entourent la réception du Rouge par les contemporains, qui concluent à un Stendhal de «mauvais ton ", sensibles qu'ils sont à la cohabitation de deux registres, la manière d'Ancien Régime voisinant avec une tonalité 1830 ; une lecture croisée qui exploite le compte rendu que Balzac a donné de la Chartreuse pour approcher «le romantisme stendhalien selon Balzac», avant d'en venir au "romantisme stendhalien selon Stendhal » tel que les brouillons de la réponse de Stendhal à Balzac le donnent à entendre, ouvrant judicieusement des aperçus sur la question du romanesque, qui fait l'objet d'un deuxième ensemble d'articles.

8 La première contribution s'intéresse au romanesque (sup)porté par le personnage (" "Peut-être", ou le roman de Julien »), ce sur quoi revient aussi la suivante, qui étend ces analyses au système des personnages secondaires dans le roman («Autour du 
personnage de Korasoff»). Plus originale, la contribution suivante envisage le romanesque comme la traduction narrative de l'arbitraire du pouvoir, libre de donner cours à «n'importe quoi » (p. 138). À ce titre, le romanesque fonctionnerait comme un marqueur de l'état politique de l'Italie du XVI ${ }^{\mathrm{e}}$ siècle («La chartreuse de Castro »). De là son moindre déploiement dans un autre récit italien, situé, lui, dans l'Italie contemporaine, La Chartreuse. L'écart manifeste dans le traitement du romanesque entre la nouvelle et le roman, tant thématiquement que stylistiquement, ouvrant sur une interprétation qui les replie l'un sur l'autre, l'énergie qui sourd du $\mathrm{XvI}^{\mathrm{e}}$ siècle révélant $a$ contrario la médiocrité du XIXe siècle (p. 145). Pierre Laforgue met en évidence l'historicité du romanesque, qui remplit toujours, chez Stendhal, une fonction éminemment poétique. La contribution suivante poursuit l'étude des rapports qu'entretiennent romanesque et narratif, l'épisode de la Fausta formant comme un isolat au sein de La Chartreuse, où éprouver, tout en les contenant aux limites de cette microstructure, les virtualités pulsionnelles du romanesque le plus débridé dont la fonction n'est pas de lâcher la bride à l'absurde mais bien de traverser les apparences pour que le récit puisse naître à sa vérité, "à la croisée de la fiction et du roman " (p.156). Le chapitre suivant surprend, qui pose que "sans entrer dans les débats complexes autant que compliqués sur la question même du réalisme, il apparaît à l'évidence que Stendhal s'en réclame avec constance et que son esthétique affichée est celle de la mimésis. (Qu'il suffise de penser à la métaphore du miroir...)»(p.161). Si Pierre Laforgue a pris, en introduction, la précaution de reconnaître des contradictions, celle-ci étonne d'autant plus qu'elle touche au cœur même de la configuration sémantique qu'il entend explorer, fragilisant ainsi son propos, et une note eût été bienvenue pour signaler au lecteur les évolutions intervenues dans la pensée de l'auteur au fil de son parcours d'exégète stendhalien. Une fois établi que « représenter la réalité, ce sera d'abord et avant tout la traduire » (p. 162), Pierre Laforgue envisage à cette aune le cas des Chroniques italiennes, données pour une traduction de manuscrits de la Renaissance alors que l'instance narrative s'en émancipe pour, fiction de la fiction, produire de la fiction, "preuve », nous dit Pierre Laforgue, " que le référent n'a aucune espèce de signification» (p.169). Le miroir revient, mais il est déformant. L'article qui conclut cette seconde partie («Dans quelle langue La Chartreuse de Parme est-elle écrite?») étend le propos à cette autre chronique italienne qui se donne, contre toute vraisemblance linguistique, pour la reproduction exacte d'archives reçues d'un chanoine de Padoue. N'ignorant pas la différence de statut entre ces textes, Pierre Laforgue s'intéresse à l'usage des italiques dans La Chartreuse, dont il fait un marqueur romanesque, l'indice d'un basculement des espaces de la narration vers ceux du roman (p. 174), instauré espace de tension (entre italique et romain, entre italien et français, entre l'Italie et la France, entre la chronique et sa "traduction", entre récit et roman...). La réponse à la question que pose le titre est résolue de façon extrêmement subtile par Pierre Laforgue qui conclut du relevé des termes en italiques à un roman parlant français mais écrit en italien, langue improbable dont le jeu explore les frontières entre le réel et ses possibles.

9 La dernière section, centrée sur les enjeux propres au roman, compte dix études. La première («Les italiques du Rouge et le Noir») poursuit l'enquête sur les italiques, singulièrement sur ce que Pierre Laforgue nomme «italiques d'énonciation » dont l'emploi manifesterait le déploiement d'un discours propre, en rupture avec la norme énonciative en vigueur dans la narration, en prise avec le sociotexte. Mais si les italiques font signe, ils ne font pas sens pour autant, aussi faut-il faire justice d'un 
supposé discours italique, autre que troué, et raccorder leur usage à un dispositif signifiant plus vaste, qui englobe avertissement, épigraphes, sous-titres et titres courants, tous ces éléments paratextuels qu'il faut penser sous les espèces de la polyphonie, facteur de rupture énonciative ou, à tout le moins, d'instabilité, rendant indécidable l'interprétation du discours idéologique qui circule dans le roman et manifeste l'obstacle opposé à la transparence du verbe. L'article suivant («La mise en texte de l'histoire dans Le Rouge et le Noir ») se penche, à partir de l'Avertissement, sur la disjonction entre les plans du récit et du roman dont Pierre Laforgue voit Stendhal jouer dans Le Rouge, interrogeant de préférence les zones de recouvrement de ces deux niveaux, et le brouillage énonciatif induit, caractérisable en termes de jeu, qui fonde la "poétique stendhalienne du roman» (p. 214), solidaire de l'instance auctoriale quand le récit l'est de l'instance narrative. Pierre Laforgue se livre à une micro-lecture très efficace pour appréhender, sous les fictions génétiques alléguées et les subterfuges énonciatifs qui prétendent nier l'historicisation du roman, sa fondamentale historicité. Suivent deux explorations de l'épisode nodal de la note secrète ( « La mise en texte de la politique dans l'épisode de la note secrète » et "Parenthèse sur une parenthèse ») où Pierre Laforgue met en évidence ce que peut le détour par la fiction pour penser une histoire qui échappe dans l'ordre du réel. «Chronologie(s) du Rouge et le Noir » dissocie la « vérité ( ?) historique » - comme eût dit le regretté Pierre Barbéris, dont les mânes sont rappelés comme est évoquée la trifonctionnalité HISTOIRE / Histoire / histoire (p. 256) - de sa textualisation, engageant le lecteur à constamment mettre le texte à l'épreuve du réel. Et vice versa. Sous les anisochronies qu'il débusque, Pierre Laforgue voit Stendhal orchestrer un gigantesque brouillage de l'histoire, qui invite à questionner son sens. Tout est fait pour désorienter le lecteur et Pierre Laforgue relève fort justement que "raconter l'histoire sous forme de chronique en ramenant les choses à leur déroulement au jour le jour a pour effet de ruiner la possibilité même d'un discours sur l'histoire» (p. 278). Ce que dit aussi l'étrange construction romanesque qui consiste à écrire une chronique de 1830 d'où s'est absentée la révolution, alors même que la chronologie retenue l'eût impliquée et alors même aussi que le souvenir de l'autre Révolution hante le roman. Vérité romanesque encore, tout entière contenue dans ce subterfuge qui escamote la révolution, devenue pure fiction, comme celle-ci a été confisquée dans le réel. Suit « Rouge, Noir et Bleu », qui tient plus lâchement à la problématique qui organise la section. «Images, signes et écriture dans La Chartreuse de Parme » renoue en revanche avec les précisions liminaires de l'ouvrage qui envisagent les effets d'un dispositif cryptographique dans le régime sémiotique de La Chartreuse. L'article suivant, "Écriture, signes et sens dans La Chartreuse de Parme ou le désenchantement du monde en 1839 » questionne la crise de la représentation à partir d'une mise en cause du langage qui, en l'absence d'un narrateur omniscient, échappe à tout "principe transcendant» (p.326) d'organisation. Il s'intéresse notamment au personnage de l'abbé Blanès pour son affinité avec le régime des signes, quand «tous les hommes ne sont plus qu'en position de lecteurs, de lecteurs qui ne veulent pas lire» (p.330). Appliquant une lecture foucaldienne, Pierre Laforgue découvre une «mutation philosophique» repérable au fait que les «signes de l'histoire » sont devenus muets et ont cessé d'être arrimés à un sens, pour ne rien dire $\mathrm{du}$ sens. Non sans quelque équilibrisme, Pierre Laforgue suit le déploiement de l'arbitraire du signe dans diverses manifestations: trucages systématiques des correspondances, billet antédaté du prince - lequel pourrait néanmoins tout autant exprimer l'arbitraire du pouvoir -, et alphabet alla monaca, qui consacre l'autonomie 
du langage et de sa notation quand message et chiffre sont donnés ensemble, preuve que "l'écriture est à elle-même son propre chiffre " (p. 337). "Le Prince moderne ou réaction et révolution dans $\mathrm{La}$ Chartreuse de Parme " poursuit l'étude de la textualisation de l'idéologie à partir des termes de « révolution » et de "république ». Exploitant les distorsions auxquelles leur mise en texte donne lieu, et suivant les chassés-croisés idéologiques des personnages qui, entre jacobinisme et ultracisme, relèvent d'une véritable perversion du sens, Pierre Laforgue conclut à la désindexation du politique et de l'idéologique et rapporte ces effets incongrus à la tentative d'acclimater dans le texte une révolution qui n'a plus de réalité que textuelle, comme le montre également la béance inscrite dans le poème de Ferrante Palla ( La ... aura-t-elle jamais une chambre et un budget? ») et la datation interpolée de 1830 dans l'Avertissement, qui convoque la référence révolutionnaire à l'horizon du texte, superposant le tour de passe-passe de Juillet au carnaval auquel se résume l'émeute parmesane qui renverse Ernest-Ranuce. Ici la fiction recouvre la vérité romanesque et, balayant l'exactitude factuelle, Pierre Laforgue montre un Stendhal à juste distance du réel de 1830, comme Hugo le sera de celui de 1848, confiant à ses Choses vues: « Février a mis une couche de république sur la France. L'ancienne société reparaît déjà dessous. Il faudra une seconde couche. À réaction révolution et demie ».

Enfin, "Son nom de Sandrino dans Parme déserte " revient sur cette défaite de l'histoire, désormais "en défaut ", et que vient maintenant relayer le roman, et le romanesque, par où passe à présent la relation au réel, le roman résidant dans «la tension entre l'historique et le romanesque » (p. 370) qui, ici encore, dit «l'énorme régression" «de l'histoire» (p.372), d'une histoire dévoyée où les pères sont les assassins de leurs enfants, par où tourne court le « roman de l'avenir ».

11 En conclusion, si le principe de composition de l'ouvrage, fruit d'un collationnement d'articles, conduit inévitablement à certaines redites, voire à l'exposé de contradictions préjudiciables à « l'effet volume », on retient de cette lecture l'attention scrupuleuse au texte qui guide Pierre Laforgue, lequel s'emploie à débusquer les normalisations abusives des éditions modernes, préalable indispensable au type de lecture qu'il met en œuvre pour donner tout leur sens aux usages stendhaliens des marqueurs graphiques (italiques, parenthèses, virgules...).

\section{AUTEURS}

\section{LAURE LÉVÊQUE}

Université de Toulon, Babel EA 2649 